\title{
Background model for the NaI(Tl) crystals in COSINE-100
}

\author{
P. Adhikari ${ }^{1}$, G. Adhikari ${ }^{1}$, E. Barbosa de Souza $^{2}$, N. Carlin $^{3}$, S. Choi ${ }^{4}$, W. Q. Choi ${ }^{5}$, M. Djamal ${ }^{6}$, A. C. Ezeribe ${ }^{7}$, \\ C. Ha ${ }^{8}$, I. S. Hahn ${ }^{9}$, A. J. F. Hubbard ${ }^{2}$, E. J. Jeon ${ }^{8, a}$, J. H. Jo ${ }^{2}$, H. W. Joo ${ }^{4}$, W. G. Kang ${ }^{8}$, M. Kauer ${ }^{11}$, W. S. Kang ${ }^{10}$, \\ B. H. Kim ${ }^{8}$, H. Kim ${ }^{8}$, H. J. Kim ${ }^{12}$, K. W. Kim ${ }^{8}$, M. C. Kim ${ }^{10}$, N. Y. Kim ${ }^{8}$, S. K. Kim ${ }^{4}$, Y. D. Kim ${ }^{8,1}$, Y. H. Kim ${ }^{8,13}$, \\ V. A. Kudryavtsev ${ }^{7}$, H. S. Lee ${ }^{8}$, J. Lee ${ }^{8}$, J. Y. Lee ${ }^{4}$, M. H. Lee ${ }^{8}$, D. S. Leonard ${ }^{8}$, W. A. Lynch ${ }^{7}$, R. H. Maruyama ${ }^{2}$, \\ F. Mouton ${ }^{7}$, S. L. Olsen ${ }^{8}$, H. K. Park ${ }^{15}$, H. S. Park ${ }^{13}$, J. S. Park ${ }^{8}$, K. S. Park ${ }^{8}$, W. Pettus ${ }^{2}$, H. Prihtiadi ${ }^{6}$, S. Ra ${ }^{8}$, \\ C. Rott $^{10}$, A. Scarff ${ }^{7}$, N. J. C. Spooner ${ }^{7}$, W. G. Thompson ${ }^{2}$, L. Yang $^{14}$, S. H. Yong ${ }^{8}$ \\ ${ }^{1}$ Department of Physics and Astronomy, Sejong University, Seoul 05006, South Korea \\ ${ }^{2}$ Wright Laboratory, Department of Physics, Yale University, New Haven, CT 06520, USA \\ ${ }^{3}$ Physics Institute, University of São Paulo, São Paulo 05508-090, Brazil \\ ${ }^{4}$ Department of Physics and Astronomy, Seoul National University, Seoul 08826, Republic of Korea \\ ${ }^{5}$ Korea Institue of Science and Technology Information, Daejeon 34141, Republic of Korea \\ ${ }^{6}$ Department of Physics, Bandung Institute of Technology, Bandung 40132, Indonesia \\ ${ }^{7}$ Department of Physics and Astronomy, University of Sheffield, Sheffield S3 7RH, UK \\ ${ }^{8}$ Center for Underground Physics, Institute for Basic Science (IBS), Daejeon 34126, Republic of Korea \\ ${ }^{9}$ Department of Science Education, Ewha Womans University, Seoul 03760, Republic of Korea \\ ${ }^{10}$ Department of Physics, Sungkyunkwan University, Seoul 16419, Republic of Korea \\ ${ }^{11}$ Department of Physics and Wisconsin IceCube Particle Astrophysics Center, University of Wisconsin-Madison, Madison, WI 53706, USA \\ 12 Department of Physics, Kyungpook National University, Daegu 41566, Republic of Korea \\ 13 Korea Research Institute of Standards and Science, Daejeon 34113, Republic of Korea \\ ${ }^{14}$ Department of Physics, University of Illinois at Urbana-Champaign,, Urbana IL 61801, USA \\ 15 Department of Accelerator Science, Graduate School, Korea University, Sejong 30019, South Korea
}

Received: 17 April 2018 / Accepted: 1 June 2018 / Published online: 12 June 2018

(C) The Author(s) 2018

\begin{abstract}
The COSINE-100 dark matter search experiment is an array of $\mathrm{NaI}(\mathrm{Tl})$ crystal detectors located in the Yangyang Underground Laboratory (Y2L). To understand measured backgrounds in the $\mathrm{NaI}(\mathrm{Tl})$ crystals we have performed Monte Carlo simulations using the Geant 4 toolkit and developed background models for each crystal that consider contributions from both internal and external sources, including cosmogenic nuclides. The background models are based on comparisons of measurement data with Monte Carlo simulations that are guided by a campaign of material assays and are used to evaluate backgrounds and identify their sources. The average background level for the six crystals $(70 \mathrm{~kg}$ total mass) that are studied is 3.5 counts/day $/ \mathrm{keV} / \mathrm{kg}$ in the (2-6) $\mathrm{keV}$ energy interval. The dominant contributors in this energy region are found to be ${ }^{210} \mathrm{~Pb}$ and ${ }^{3} \mathrm{H}$.
\end{abstract}

\section{Introduction}

COSINE-100 is a dark matter search experiment consisting of a $106 \mathrm{~kg}$ array of eight ultra-pure $\mathrm{NaI}(\mathrm{Tl})$ crystals $[1,2]$.

\footnotetext{
a e-mail: ejjeon@ibs.re.kr
}

Its primary goal is to test DAMA/LIBRA's assertion of an observation of annual modulation signal [3-6]. The experiment has been operating at the Yangyang Underground Laboratory (Y2L) since September 2016 [7]. COSINE-100 is one of several $\mathrm{NaI}(\mathrm{Tl})$-based dark matter searches in operation (DM-Ice17 [8], ANAIS [9,10]) or under development (DM-Ice [11], KamLAND-PICO [12], SABRE [13], COSINUS [14]). Previously, the KIMS-CsI experiment put a limit on interaction rates in CsI crystals $[15,16]$ that precluded the interpretation of the DAMA modulation signal as being due to WIMPs scattering from I or Tl nuclei, considering the different quenching factors of iodine and thallium for $\mathrm{NaI}(\mathrm{Tl})$ and $\operatorname{CsI}(\mathrm{Tl})$.

The COSINE-100 crystal array is immersed in a tank of liquid scintillator (LS) that tags backgrounds that originate from outside the $\mathrm{LS}$ as well as decays of ${ }^{40} \mathrm{~K}$ nuclides inside the crystals. To determine the sources of the backgrounds, we have performed Monte Carlo simulations using the Geant4 toolkit (V.4.9.6.p02) [17] and built a background model for the eight detectors by iteratively fitting their contributions to the measured energy spectra; two crystals are excluded in this paper due to their low light yields, which result in a background spectrum without characteristic peaks of iso- 
topes by the worse energy resolution, and relatively higher background contamination in the low energy region.

The paper is structured as follows: the COSINE-100 experimental setup is described in Sect. 2 . Section 3 describes the background modeling, with the simulation method described in Sect. 3.2, sources of the background internal and external to the crystal and of cosmogenic origin in Sects. 3.23.4. Section 4 describes the comparison and fit to the data, and Sect. 5 provides discussions of the background developed by the fits. Finally Sect. 6 provides conclusions.

\section{COSINE-100 setup and simulation geometry}

The experimental setup is described in detail in Ref. [7]. The simplified geometry used for the simulations is shown in Fig. 1. Eight $\mathrm{NaI}(\mathrm{Tl})$ crystals, arranged in two layers, are located in the middle of a four-layer shielding structure. From outside inward, the four shielding layers are plastic scintillator panels, a lead-brick castle, a copper box, and a scintillating liquid. The eight $\mathrm{NaI}(\mathrm{Tl})$ crystal assemblies and their support table are immersed in the scintillating liquid that serves both as an active veto and a passive shield.

The eight $\mathrm{NaI}(\mathrm{Tl})$ crystals were grown out of batches of powder provided by Alpha Spectra [18] with successive improvements. The first attempts, which produced an order of magnitude reduction in ${ }^{40} \mathrm{~K}$, were AS-B and AS-C. This was followed by WIMPScint-II (AS-WSII) which reduced the ${ }^{210} \mathrm{~Pb}$ contamination, and WIMPScint-III (AS-WSIII) which resulted in another factor of two reduction of ${ }^{40} \mathrm{~K}$. The results are summarized in Table 1 . The final crystals are cylindrically shaped and hermetically encased in OFE copper tubes with wall thickness of $1.5 \mathrm{~mm}$ and quartz windows (12.0 mm thick) at each end. Each crystal's lateral surfaces were wrapped in roughly 10 layers of $250 \mu \mathrm{m}$-thick PTFE reflective sheets. The quartz windows are optically coupled to each end of the crystal via $1.5 \mathrm{~mm}$ thick optical pads. These, in turn, are optically coupled to 3-inch Hamamatsu R12669SEL photomultiplier tubes (PMTs) with a thin layer of high viscosity optical gel. The PMTs are protected from the liquid scintillator by a housing made of copper and PTFE.

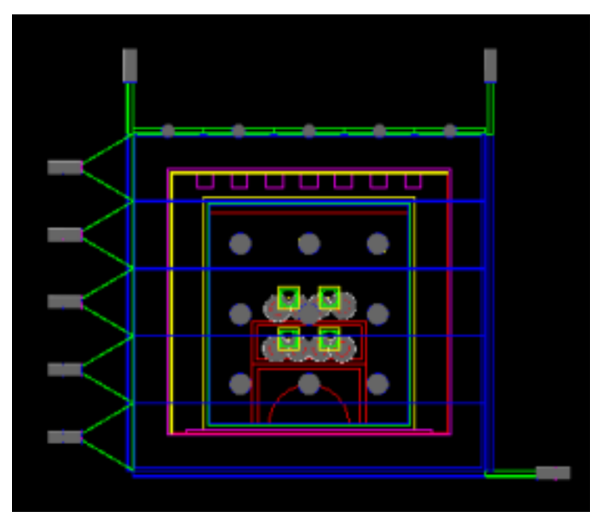

(a) Front view

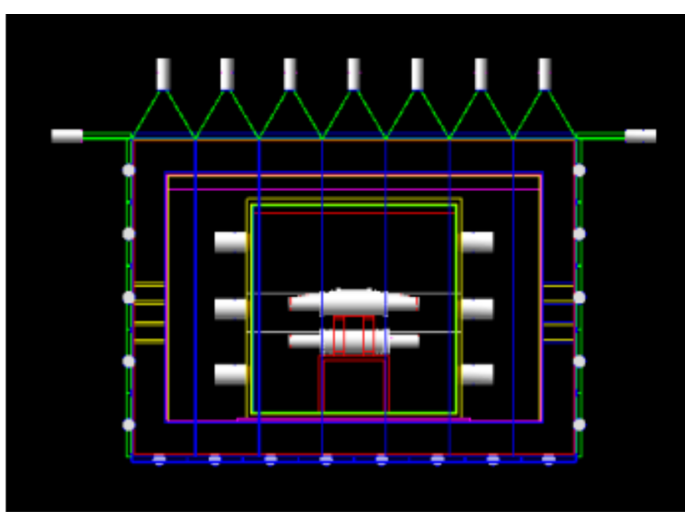

(b) Side view

Fig. 1 Front (a) and Side (b) views of the detector geometry used in the Geant4 simulations. From outside inward, the four shielding layers include: $3 \mathrm{~cm}$ thick plastic scintillator panels (dark blue), $20 \mathrm{~cm}$ lead (pink), $3 \mathrm{~cm}$ copper box (light green), and liquid scintillator (not shown)

Table 1 Radioactive contamination levels in the COSINE-100 crystals reported in Ref. [7]. These were used as inputs when fitting the simulations to the data. The ${ }^{238} \mathrm{U}$ and ${ }^{232} \mathrm{Th}$ decays were assumed to be in equilibrium

\begin{tabular}{lllllll}
\hline Crystal & $\begin{array}{r}\text { Mass } \\
(\mathrm{kg})\end{array}$ & $\begin{array}{l}\text { Size (inches) } \\
(\text { diameter } \times \text { length) }\end{array}$ & Powder & $\begin{array}{l}\alpha \text { Rate } \\
(\mathrm{mBq} / \mathrm{kg})\end{array}$ & $\begin{array}{l}{ }^{40} \mathrm{~K} \\
(\mathrm{ppb})\end{array}$ & $\begin{array}{l}{ }^{238} \mathrm{U} \\
(\mathrm{ppt})\end{array}$ \\
\hline Crystal-1 & 8.3 & $5.0 \times 7.0$ & AS-B & $3.20 \pm 0.08$ & $34.7 \pm 4.7$ & $<0.02$ \\
Crystal-2 & 9.2 & $4.2 \times 11.0$ & AS-C & $2.06 \pm 0.06$ & $60.6 \pm 4.7$ & $<0.12$ \\
Crystal-3 & 9.2 & $4.2 \times 11.0$ & AS-WSII & $0.76 \pm 0.02$ & $34.3 \pm 3.1$ & $<0.04$ \\
Crystal-4 & 18.0 & $5.0 \times 15.3$ & AS-WSII & $0.74 \pm 0.02$ & $33.3 \pm 3.5$ & $0.4 \pm 0.2$ \\
Crystal-5 & 18.3 & $5.0 \times 15.5$ & AS-C & $2.06 \pm 0.05$ & $82.3 \pm 5.5$ & $<0.3$ \\
Crystal-6 & 12.5 & $4.8 \times 11.8$ & AS-WSIII & $1.52 \pm 0.04$ & $16.8 \pm 2.5$ & $<0.02$ \\
Crystal-7 & 12.5 & $4.8 \times 11.8$ & AS-WSIII & $1.54 \pm 0.04$ & $18.7 \pm 2.8$ & $0.6 \pm 0.2$ \\
Crystal-8 & 18.3 & $5.0 \times 15.5$ & AS-C & $2.05 \pm 0.05$ & $54.3 \pm 3.8$ & $<0.6$ \\
\hline
\end{tabular}


The following components of the detector have been included in the simulation: PTFE reflective sheets, copper tubes, quartz windows, optical gel, PMT housing, and PMTs.

\section{Background modeling}

\subsection{Simulation method}

The Physics list classes of G4EmLivermorePhysics for low energy electromagnetic process and G4RadioactiveDecay for radioactive decay process were used [19-21]. The ${ }^{238} \mathrm{U}$ and ${ }^{232} \mathrm{Th}$ decay chains were treated as broken at the longlived parts of the chain. The ${ }^{238} \mathrm{U}$ chain was broken into five distinct groups and the ${ }^{232} \mathrm{Th}$ chain was broken into three groups. The details are reported in Ref. [22].

Each simulated event record includes all energy deposited in the crystals within an event window of $10 \mu$ s from the time a decay is generated, to account for the conditions in the data acquisition system (DAQ) of the experimental setup [7]. Sometimes decays with relatively short half-lives, such as ${ }^{212}$ Po decay (with a half-life of $300 \mathrm{~ns}$ ) and the subsequent daughter decays will appear in the $10 \mu$ s time window, resulting in pileup events. They are treated as a single event in the simulation.

The simulated spectrum was convolved with an energy dependent energy resolution function developed during a calibration run. Calibration points were measured using $\gamma$-ray sources: $59.5 \mathrm{keV}\left({ }^{241} \mathrm{Am}\right), 1173.2$ and $1332.5 \mathrm{keV}\left({ }^{60} \mathrm{Co}\right)$. Internal background peaks at 3.2 and $1460.8 \mathrm{keV}$ from ${ }^{40} \mathrm{~K}$, $67.3 \mathrm{keV}$ from ${ }^{125} \mathrm{I}$, and $609.3 \mathrm{keV}$ from ${ }^{214} \mathrm{Bi}$ were used to calibrate the measured spectra; peaks at 3.2, 59.5, and $67.3 \mathrm{keV}$ were used for the low energy calibration below $70 \mathrm{keV}$.

\subsection{Internal backgrounds in the $\mathrm{NaI}(\mathrm{Tl})$ crystals}

After the insertion of the crystals into the shield and prior to filling the liquid scintillator container, their background levels were measured to verify that they were free of any additional contamination. Overall, the eight crystals have acceptable ${ }^{238} \mathrm{U}$ and ${ }^{232} \mathrm{Th}$ contaminations as shown in Table 1 [7]. Secular equilibrium in the chains is assumed for the interpretation of ${ }^{238} \mathrm{U}$ and ${ }^{232} \mathrm{Th}$ related radioactivity measurements, with the exception of ${ }^{210} \mathrm{~Pb}$.

In order to estimate the background contributions from ${ }^{238} \mathrm{U},{ }^{232} \mathrm{Th},{ }^{40} \mathrm{~K}$, and ${ }^{210} \mathrm{~Pb}$, we simulated background spectra from the internal radioactive contaminants and normalized them by their measured activities in Table 1 . In the normalization we assumed a chain equilibrium and, thus, all related activities within the chains are equal to the ${ }^{238} \mathrm{U}$, ${ }^{232} \mathrm{Th}$, and ${ }^{40} \mathrm{~K}$ activities multiplied by the branching ratios for decay of the daughter isotopes. We also added the back- ground simulation of internal ${ }^{210} \mathrm{~Pb}$ by considering the measured $\alpha$ rate. The resultant background contributions, except for those from ${ }^{40} \mathrm{~K}$ and ${ }^{210} \mathrm{~Pb}$, were negligible in all eight crystals.

The ${ }^{40} \mathrm{~K}$ contribution is reduced by the LS veto detector. To measure the reduction efficiency of the ${ }^{40} \mathrm{~K}$ generated $3.2 \mathrm{keV}$ emission background provided by tagging the accompanying $1460.8 \mathrm{keV} \gamma$-ray in one of the other $\mathrm{NaI}(\mathrm{Tl})$ crystals or the LS, and to compare this to the efficiency provided by the other crystals alone, we generated ${ }^{40} \mathrm{~K}$ decays at random locations inside a $\mathrm{NaI}(\mathrm{Tl})$ crystal for the cases with and without the LS veto. From these simulations, we determined that the Crystal- 6 tagging efficiency by other crystals without LS is $31.7 \pm 0.1 \%$ and by the LS only is $64.9 \pm 0.2 \%$. The total combined efficiency is $81.7 \pm 0.3 \%$. The efficiency is measured in the crystal energy range between 2 and $6 \mathrm{keV}$ by requiring the LS energy deposit be larger than $20 \mathrm{keV}$. Efficiencies vary depending on the crystal location in the detector. For example, Crystal- 1 (at the corner of the $4 \times 2$ array) shows higher coverage by the LS $(75 \%)$ than neighboring crystals (17\%), but the combined efficiency is similar to that of Crystal-6 (82\%). The tagging efficiency of the $1460.8 \mathrm{keV}$ $\gamma$-ray in the LS-only case is lower because the range of the $\gamma$-ray in the $\mathrm{NaI}(\mathrm{Tl})$ crystal is shorter than in the LS. Therefore, more $\gamma$-rays are stopped in the other crystals than in the LS. These estimated efficiencies are in agreement with measurements [7]. Accordingly, the ${ }^{40} \mathrm{~K}$ background level is reduced by as much as $80 \%$ by requiring single-hit crystal events with no signal in the LS.

The ${ }^{210} \mathrm{~Pb}$ contribution is estimated by modeling the background from bulk ${ }^{210} \mathrm{~Pb}$ and surface ${ }^{210} \mathrm{~Pb}$ as discussed in Sect. 4.

\subsection{External background sources}

The external $\gamma$ background from the radioactive isotopes in the surrounding rocks is shielded by the $20 \mathrm{~cm}$-thick lead castle and the $3 \mathrm{~cm}$-thick copper box. By using the full shielding structure with $N_{2}$ gas flowing into the inside of the copper shield to avoid backgrounds from ${ }^{222} \mathrm{Rn}$ in the air at $\mathrm{Y} 2 \mathrm{~L}$ (measured to be $1.20 \pm 0.49 \mathrm{pCi} / \mathrm{L}$ [23]), we reduced the environmental background by a factor of 10,000 based on the measurements of a high-purity Ge (HPGe) detector, thus ensuring that those contributions are negligibly small.

Despite all the efforts to block backgrounds due to external sources, some backgrounds from radioactive contaminations in detector components inside the shielding are still expected, including from the PMTs, grease, copper case, bolts, cables, acrylic supports, liquid scintillator, copper box, and steel that supports the lead block housing. We simulated background spectra from those external sources to test their effects and compared the shapes of contributions to the crystals' energy spectra. We found that all the spectra from these external 
Table 2 Radioactivity levels in detector components inside the shielding. (a) The radioactivities were measured with a HPGe detector at Y2L; upper limits are quoted with $90 \%$ C.L. The PMTs are measured in units of $\mathrm{mBq} / \mathrm{PMT}$ and the other external sources are measured in units of $\mathrm{mBq} / \mathrm{kg}$ (b) SEL means "selected for high quantum efficiency"

\begin{tabular}{|c|c|c|c|}
\hline \multirow[t]{2}{*}{ External source } & \multicolumn{3}{|c|}{ Radioactivity $^{a}$} \\
\hline & $\mathrm{U}\left({ }^{214} \mathrm{Bi}\right)$ & Th $\left({ }^{228} \mathrm{Ac}\right)$ & $\left({ }^{40} \mathrm{~K}\right)$ \\
\hline $\begin{array}{l}\text { PMT [1] } \\
\left(\mathrm{R}_{\left.12669 \mathrm{SEL}^{b}\right)}\right.\end{array}$ & $25 \pm 5$ & $12 \pm 5$ & $58 \pm 5$ \\
\hline Quartz window & $<1.8$ & $<7.5$ & $<20$ \\
\hline PTFE reflector & $<0.5$ & $<1.0$ & $<6.4$ \\
\hline Cable ties & $<4.2$ & $<3.5$ & $149 \pm 32$ \\
\hline LS & $<2.7$ & $<3.3$ & $7 \pm 4$ \\
\hline
\end{tabular}

sources are similar in shape and, thus, could be represented by a spectrum that is obtained by simulating ${ }^{238} \mathrm{U},{ }^{232} \mathrm{Th}$, and ${ }^{40} \mathrm{~K}$, distributed randomly in the volume outside the eight crystals. Because the PMTs are the main contributer to the external background we used two kinds of spectra for the external background modeling; one is the spectrum from the PMTs and another is the spectrum from the other external sources that is treated as a parameter floating in the fit. The radioactivity levels of the PMTs and PMT surrounding parts were measured underground with a HPGe detector and the results are listed in Table 2 . We used the measured activities from the PMTs to constrain the data fitting and treated background contributions from the PMTs in nine groups as broken at the long-lived parts of the chain.

\subsection{Treatment of cosmogenic radionuclides}

Although the eight $\mathrm{NaI}(\mathrm{Tl})$ crystals had underground radioactivity cooling times that ranged from several months to three years, there are still background contributions due to the longlived cosmogenic isotopes that were activated by cosmic rays while they were on the surface.

To consider these backgrounds, we first checked the list of cosmogenic radioactive isotopes that are produced in $\mathrm{NaI}(\mathrm{Tl})$, as reported in Refs. [24-27]. In Table 3a, we list the contributing cosmogenic isotopes with their half lives; shortlived isotopes, for which half lives are less than a year, are ${ }^{125} \mathrm{I},{ }^{121} \mathrm{Te},{ }^{121 m} \mathrm{Te},{ }^{123 m} \mathrm{Te},{ }^{125 m} \mathrm{Te},{ }^{127 m} \mathrm{Te}$, and ${ }^{113} \mathrm{Sn}$ and long-lived isotopes are ${ }^{109} \mathrm{Cd},{ }^{3} \mathrm{H}$, and ${ }^{22} \mathrm{Na}$. The radioactivity cooling time at $\mathrm{Y} 2 \mathrm{~L}$ for each crystal at the time datataking for COSINE-100 started, is listed in Table 3b. The short-lived isotopes are not expected to contribute to either Crystal-1 or Crystal-2 because their cooling times are long enough to reduce these activities to a negligible level.

However, we expect some backgrounds from the shortlived isotopes in other crystals because their production rates at sea level, as listed in Table 3a, are high and their cooling
Table 3 Cosmogenic radionuclides in $\mathrm{NaI}(\mathrm{Tl})$ crystal (a) and exposure time and radioactivity cooling time at Y2L (b)

\begin{tabular}{lll}
\hline $\begin{array}{l}\text { Cosmogenic } \\
\text { isotopes }\end{array}$ & $\begin{array}{l}\text { Half-life } \\
\text { (days) }\end{array}$ & $\begin{array}{l}\text { Production rate at } \\
\text { sea level [24] } \\
\text { (counts/kg/day) }\end{array}$ \\
\hline (a) & 59.4 & 221 \\
${ }^{125} \mathrm{I}$ & 19.17 & 93 \\
${ }^{121} \mathrm{Te}$ & 164.2 & 93 \\
${ }^{121 m} \mathrm{Te}$ & 119.2 & 52 \\
${ }^{123 m} \mathrm{Te}$ & 57.4 & 74 \\
${ }^{125 m} \mathrm{Te}$ & 106.1 & 93 \\
${ }^{127 m} \mathrm{Te}$ & 115.1 & 9.0 \\
${ }^{113} \mathrm{Sn}$ & 461.4 & 4.8 \\
${ }^{109} \mathrm{Cd}$ & 4500 & 26 \\
${ }^{3} \mathrm{H}$ & 951 & 66 \\
${ }^{22} \mathrm{Na}$ & Exposure time & Radioactivity \\
\hline $\mathrm{Crystal}$ & (see text) (years) & cooling time at Y2L \\
& & (years)
\end{tabular}

(b)

$\begin{array}{lll}\text { Crystal-1 } & 2 & 3 \\ \text { Crystal-2 } & 0.75 & 2.75 \\ \text { Crystal-3 } & & 1.2 \\ \text { Crystal-4 } & 1.7 & 0.5 \\ \text { Crystal-6 } & 0.3 & 0.6 \\ \text { Crystal-7 } & 0.3 & 0.6\end{array}$

times are less than or equal to a year. In addition, there are long-lived ${ }^{109} \mathrm{Cd},{ }^{3} \mathrm{H}$, and ${ }^{22} \mathrm{Na}$ nuclides that are potentially hazardous background sources; $e$.g., the beta-decay spectrum of tritium has an endpoint energy of $18 \mathrm{keV}$. We thus need to understand their background contributions in the low energy region, especially in the (2-6) keV WIMP signal region of interest (ROI). Because it is impossible to compute the initial activities of those isotopes from the production rates in each crystal at Y2L without knowing the cosmic ray exposure conditions: time, location, altitude, etc. [24], we investigated the correlation of characteristic peaks produced by $\gamma / \mathrm{X}$-rays from the decay of cosmogenic isotopes.

- ${ }^{109} \mathrm{Cd}$ decays by electron capture to the isomeric state of ${ }^{109} \mathrm{Ag}$ depositing in the crystal the binding energy of the Ag K-shell electrons $(25.5 \mathrm{keV})$, that will be accompanied by the $88 \mathrm{keV} \gamma$ ray from the isomer transition of ${ }^{109} \mathrm{Ag}$ having a mean time of $57.4 \mathrm{~s}$. By using the timing information of two adjacent events that have each $25.5 \mathrm{keV}$ and $88 \mathrm{keV}$, we measured the background contribution of ${ }^{109} \mathrm{Cd}$ in Crystal-4 and found it to be $0.10 \pm 0.01 \mathrm{mBq} / \mathrm{kg}$. 
- ${ }^{22} \mathrm{Na}$ decays via positron emission $(90 \%)$ and electron capture (10\%), followed by $1274.6 \mathrm{keV} \gamma$-ray emission with a mean lifetime of $3.8 \mathrm{yr}$. The electron capture decay produces $0.9 \mathrm{keV}$ emissions. Therefore, $\sim 10 \%$ of the ${ }^{22} \mathrm{Na}$ decay will produce $0.9 \mathrm{keV} \mathrm{X}$-rays and $1274.6 \mathrm{keV}$ $\gamma$ rays simultaneously. Meanwhile, the positron will be converted to two $511 \mathrm{keV}$ annihilation $\gamma$ rays.

However, it is generally difficult to measure long-lived cosmogenics' activities, such as those for ${ }^{3} \mathrm{H}$, directly from the data due to their long half-lives. Therefore, we simulated background spectra from cosmogenic isotopes listed in Table $3 \mathrm{a}$ and used their shapes in the data fitting, while floating their unknown fractions. The details of their treatment in the background model for each $\mathrm{NaI}(\mathrm{Tl})$ crystal are discussed in Sect. 4.

\section{Comparison with measured data}

To model the COSINE-100 detector backgrounds, we used data collected between Oct 21 and Dec 19 2016. We applied low energy noise cuts as described in Ref. [7].

The LS veto threshold was set at $20 \mathrm{keV}$ for both single crystal and multiple crystal events. Events in coincidence with an LS signal ( $>20 \mathrm{keV}$ in LS) and/or more than 4 photo electrons in other crystals are defined as multiple events.

Crystals 1 and 2 have no short-lived cosmogenic contaminants and were used for comparisons. Since Crystal-3 and Crystal-4 have different production times and delivery times at the Y2L, their expected short-lived cosmogenic activities are quite different. But they are made of the same NaI powder and expected to have similar internal activities. Crystal-6 and Crystal-7 are twin crystals made up of the same $\mathrm{NaI}$ powder and at the same time. They are installed between Crystal-5 and 8 and expected to have similar external background. Both crystals are expected to have the same amount of internal, external and cosmogenic activities. We compare the activities of these crystal subdivisions.

We use a log likelihood method to fit the data. The fitting range is $6 \mathrm{keV}-2 \mathrm{MeV}$ and we perform four simultaneous fits: single-hit low-energy, single-hit high-energy, multiplehit low-energy and multiple-hit high-energy. Because different FADC systems are used for low- and high-energy data the resolutions are different. Low-energy means below $70 \mathrm{keV}$ and high-energy means above $70 \mathrm{keV}$. The internal ${ }^{238} \mathrm{U}$, ${ }^{232} \mathrm{Th}$, and ${ }^{40} \mathrm{~K}$ levels are constrained to be within $20 \%$ of their measured values. We use a $10 \mu \mathrm{m}$ thickness to distinguish between surface and bulk ${ }^{210} \mathrm{~Pb}$ components to study surface contamination in the crystals that is generated uniformly within the thickness; their levels are allowed to float. The ${ }^{238} \mathrm{U},{ }^{232} \mathrm{Th}$, and ${ }^{40} \mathrm{~K}$ levels in the PMTs are constrained to be within $50 \%$ of their measurements, taking into account contributions from PMT surrounding parts. Cosmogenic and the external ${ }^{238} \mathrm{U},{ }^{232} \mathrm{Th},{ }^{40} \mathrm{~K}$, and ${ }^{60} \mathrm{Co}$ levels from other parts of the detector are free floated.

\subsection{Background model for crystals 1 and 2}

The surface exposure time of Crystal-1 is longer than Crystal2 by more than a year and it is expected to have more longlived cosmogenic isotopes such as ${ }^{3} \mathrm{H}$ and ${ }^{109} \mathrm{Cd}$. In addition, it was delivered by air while Crystal-2 was shipped by sea. At first we did not consider long-lived cosmogenic isotopes in the data fitting for Crystal-1 and the resulting four-channel fits are shown in Fig. 2. The overall energy spectrum is well matched to the data for both single-hit and multiple-hit events, except for the single-hit low-energy events. The agreement between the measured and fitted multiple-hit background spectra of Crystal-2, 3, 4, 6, and 7 is as good as shown for Crystal-1.

The peak around $46 \mathrm{keV}$ is due to ${ }^{210} \mathrm{~Pb}$ in the crystals. However only a small part, $4.3 \%$, is due to the $46.5 \mathrm{keV}$ gamma-ray line; most of the events in the peak are from the conversion electrons, Auger electrons, and X-rays, followed by beta electrons from the decay to ${ }^{210} \mathrm{Bi}$. As a result, the peak is non-Gaussian. It is not well reproduced by the simulations using the Geant 4 version 4.9.6.p02 and, thus, we will consider a higher version number for further modeling.

We found that it was not possible to model the Crystal-1 and 2 background spectra for energies below $30 \mathrm{keV}$ with only bulk and surface ${ }^{210} \mathrm{~Pb}$ contaminations (see Fig. 2a). To get adequate fits to both crystals, we had to include significant contributions from ${ }^{3} \mathrm{H}$ and ${ }^{109} \mathrm{Cd}$, as shown in Fig. 3a, b. They are also included in the models for the other crystals.

Internal ${ }^{210} \mathrm{~Pb}$ contamination levels independently determined from the alpha activities in the crystals are listed in Table 1. To study surface contamination in the crystals both bulk and surface ${ }^{210} \mathrm{~Pb}$ components are free floated in the fit. The ${ }^{40} \mathrm{~K}$ contamination levels in the crystals are identified by coincident signals between a $3.2 \mathrm{keV}$ emission in one $\mathrm{NaI}(\mathrm{Tl})$ detector and a $1460.8 \mathrm{keV}$ gamma-ray in one of the other $\mathrm{NaI}(\mathrm{Tl})$ crystals or an energy deposition in the LS. Background from the readout PMTs and surrounding material is considered as external components.

\subsection{Background model for crystals 3 and 4}

Although Crystal-3 and 4 were both grown with WIMPScint-II grade powder by Alpha Spectra in July 2014, they were delivered to the $\mathrm{Y} 2 \mathrm{~L}$ at different times. Crystal-3 has a complicated exposure history and was repaired once before arriving at Y2L in July 2015 and has remained underground ever since. On the other hand, Crystal-4 was delivered at the Y2L in March 2016 after being exposed to surface-level cosmic rays for about 2 years and was installed for COSINE- 


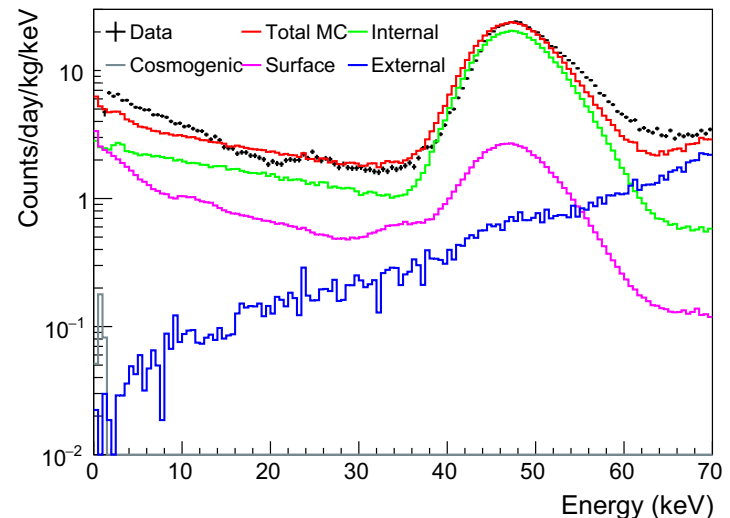

(a) Single-hit events with low-energy data

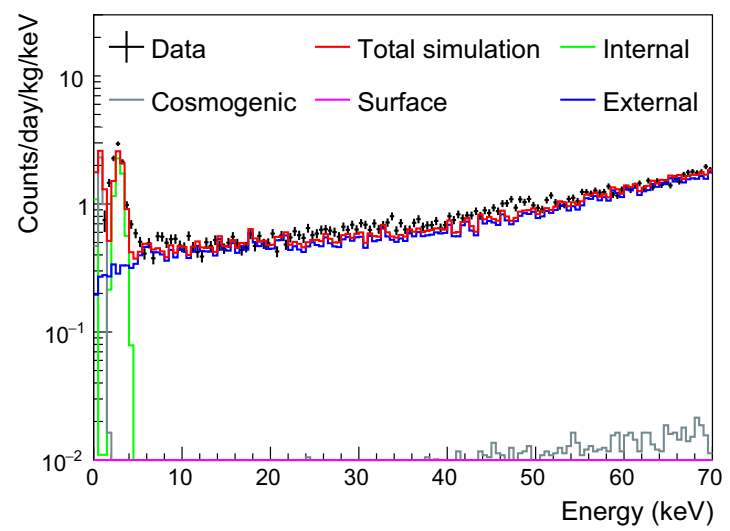

(c) Multiple-hit events with low-energy data

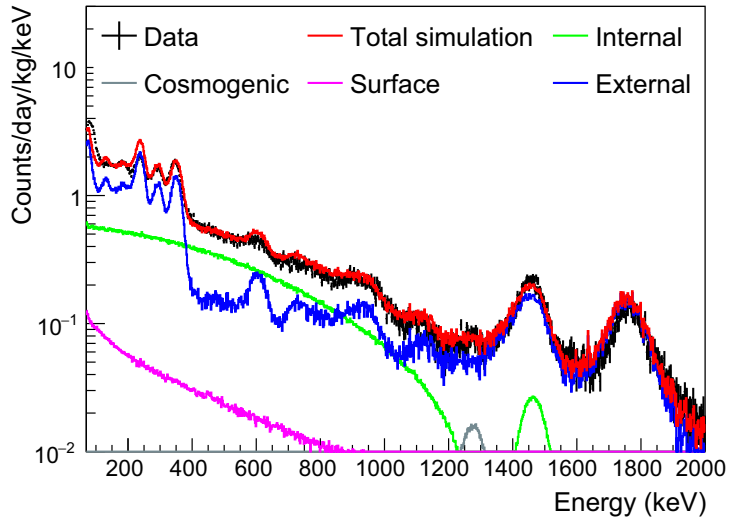

(b) Single-hit events with high-energy data

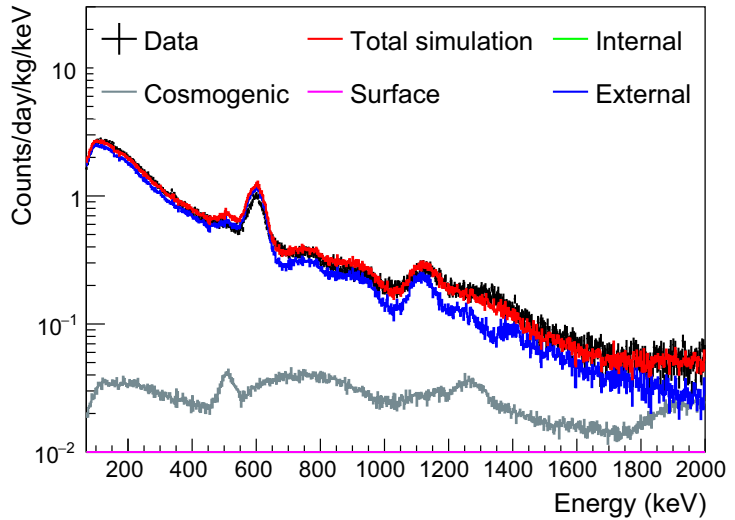

(d) Multiple-hit events with high-energy data

Fig. 2 Measured single- and multiple-hit background spectra of Crystal-1 fitted with all simulated background spectra using four channel simultaneous fitting. ${ }^{3} \mathrm{H}$ and ${ }^{109} \mathrm{Cd}$ are excluded in the fit

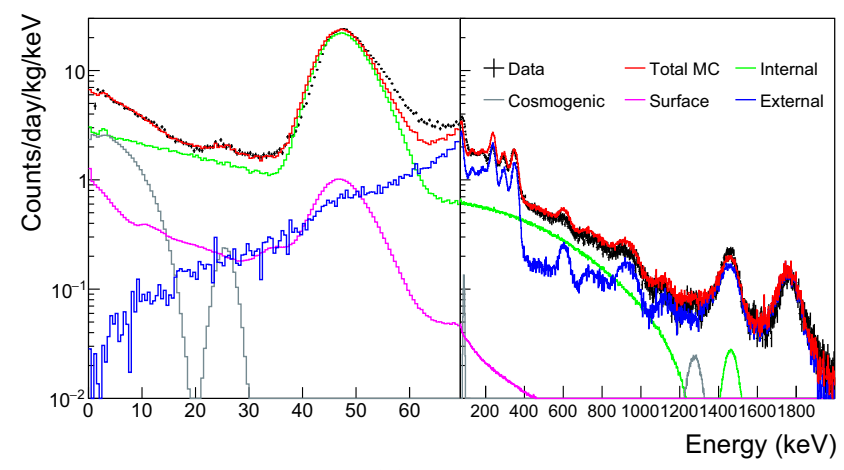

(a) Crystal-1

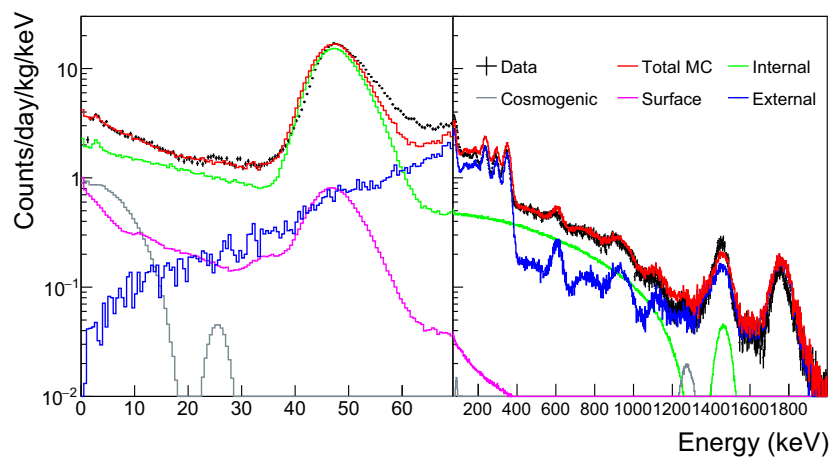

(b) Crystal-2

Fig. 3 Measured single-hit background spectra of Crystal-1 and Crystal-2 fitted with all simulated background spectra. ${ }^{3} \mathrm{H}$ and ${ }^{109} \mathrm{Cd}$ are included in the fit

100 after just six months of cooling. As a result, Crystal-4 is expected to have short-lived cosmogenic isotopes as well as long-lived cosmogenic isotopes.

The background spectrum of Crystal-3, shown in Fig. 4a, has a peak around $10 \mathrm{keV}$ that has not changed over time. To understand its origin, we studied the effect of surface ${ }^{210} \mathrm{~Pb}$ from PTFE reflective sheets that wrapped each crystal's lat- eral surfaces in ten layers with $250 \mu \mathrm{m}$ total thickness. We simulated the background spectrum of ${ }^{210} \mathrm{~Pb}$ by generating it randomly within the layer of the PTFE sheets with various thicknesses: $0.1,0.5,1.0,2.0,3.0,5.0 \mu \mathrm{m}$, and also in the bulk. The simulated spectra are shown in Fig. 5, where each color represents the different surface depths and the bulk reflector (black solid line). The peaks at $12 \mathrm{keV}$ and $46 \mathrm{keV}$, 


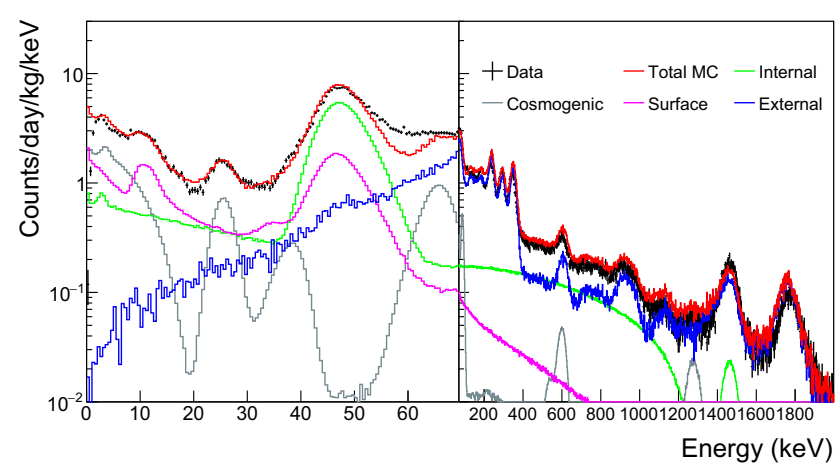

(a) Crystal-3

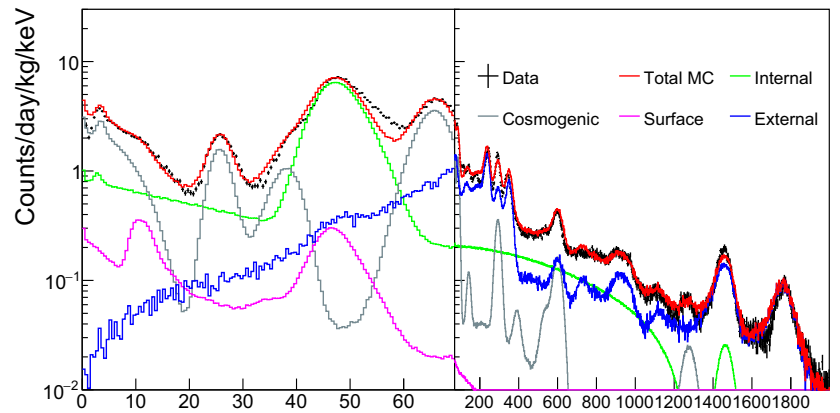

Energy (keV)

(b) Crystal-4

Fig. 4 Measured single-hit background spectra of Crystal-3 and Crystal-4 fitted with all simulated background spectra

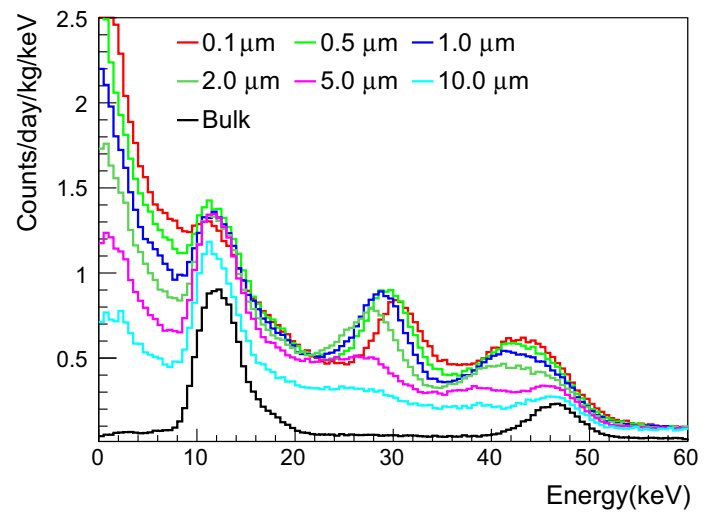

Fig. 5 Comparison of background spectra of ${ }^{210} \mathrm{~Pb}$ simulated for various surface thicknesses of PTFE reflector. The activity of $1 \mathrm{mBq} / \mathrm{kg}$ is used to normalize the simulation results

which are prominent for the bulk reflector, are due to the $\mathrm{X}$-rays and $46.5 \mathrm{keV} \gamma$-ray from the decays of ${ }^{210} \mathrm{~Pb}$, respectively. Conversion electrons contribute peaks around $30 \mathrm{keV}$ and $43 \mathrm{keV}$ and beta electrons contribute a continuum at peaks at low energy. Since the conversion electrons' energy losses depend on the thickness of PTFE that they penetrate, these peaks move to lower energies as the depth increases. In the simulation we used the spectrum of ${ }^{210} \mathrm{~Pb}$ from the bulk

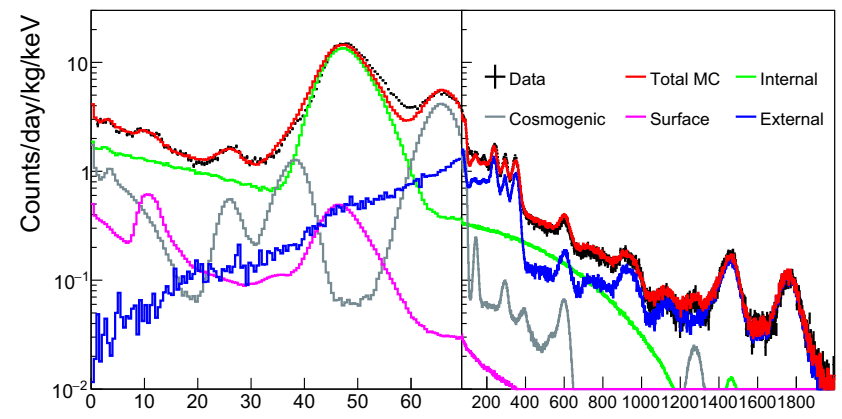

(a) Crystal-6 reflector to model the $12 \mathrm{keV}$ peak because the surfaces can be treated as a bulk with $\sim 10$ layers of PTFE sheets.

The Crystal-4 spectrum, shown in Fig. 4b, has three correlated peaks from the decay of ${ }^{109} \mathrm{Cd}: \sim 3$ and $\sim 25 \mathrm{keV}$ binding energies from L-shell/K-shell electron captures and $\sim 88 \mathrm{keV}$ gamma-ray line from the isomer transition of ${ }^{109} \mathrm{Ag}$. We also determined its half-life by measuring the change of $\sim 25 \mathrm{keV}$ binding energies contribution over time with a result that is in a good agreement with the 462 day expectation. The activity from the fit to the data, found to be $0.09 \pm 0.06 \mathrm{mBq} / \mathrm{kg}$, is consistent with the measurement.

\subsection{Background model for crystals 6 and 7}

Crystal-6 and 7 are twin crystals made from WIMPScintIII grade powder by Alpha Spectra at the same time. They were installed 7 months after their delivery to $\mathrm{Y} 2 \mathrm{~L}$, similar to Crystal-4. However their surface exposure times were shorter than those of the other crystals. Figure 6 shows that the fitted simulation spectra accurately reproduce the measured data. As expected, they have similar contamination levels of shortlived cosmogenic isotopes.

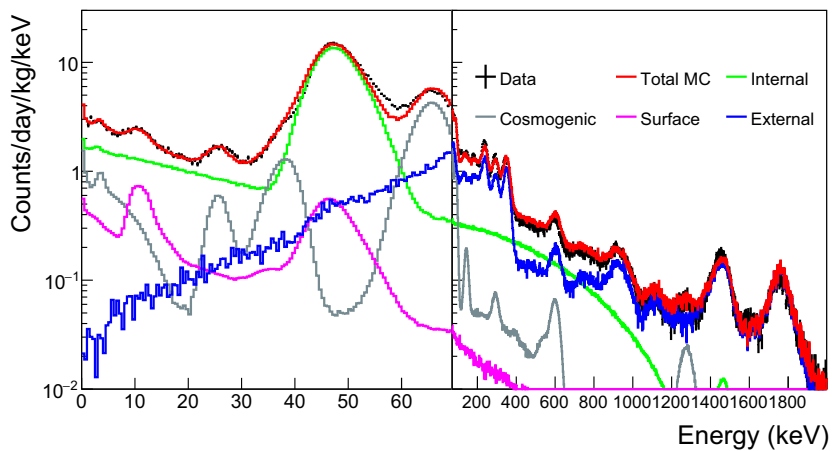

(b) Crystal-7
Energy (keV)

Fig. 6 Measured single-hit background spectra of Crystal-6 and Crystal-7 fitted with all simulated background spectra 


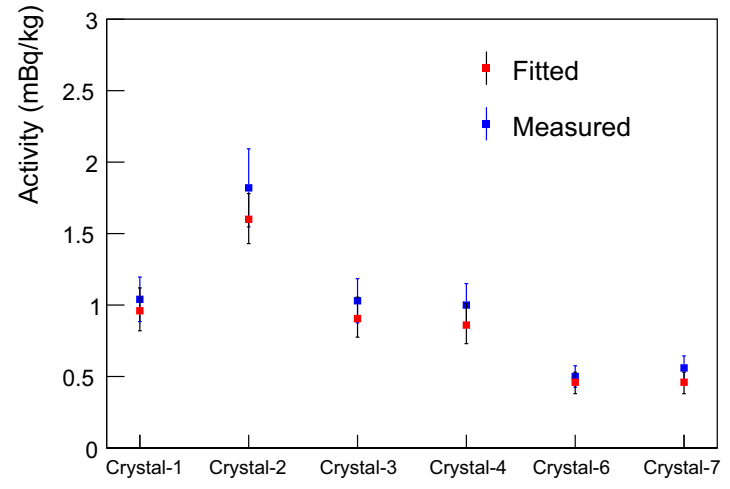

(a) Internal ${ }^{40} \mathrm{~K}$

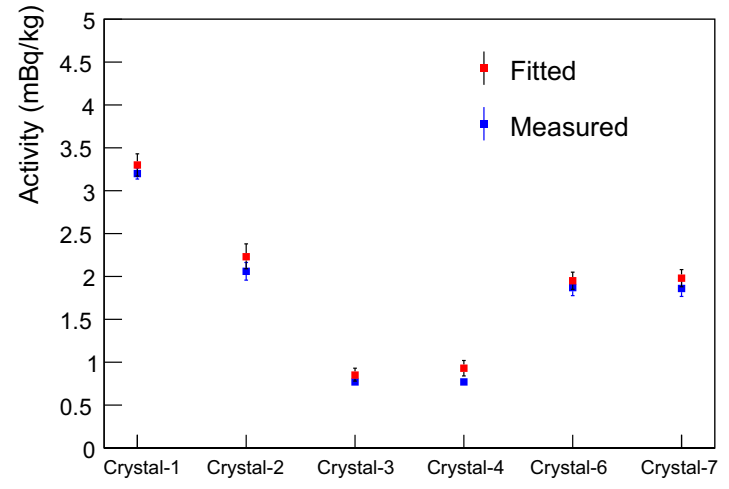

(b) Bulk ${ }^{210} \mathrm{~Pb}$

Fig. 7 Comparison of the measured and the fitted activity levels in six $\mathrm{NaI}(\mathrm{Tl})$ crystals

\section{Discussion of fitted background spectra}

We have simulated background spectra from radioactive sources: full decay chains of ${ }^{238} \mathrm{U},{ }^{232} \mathrm{Th}$, and ${ }^{40} \mathrm{~K}$ from the crystals, 16 PMTs, and the other external sources; bulk and surface ${ }^{210} \mathrm{~Pb}$ from the crystals; surface ${ }^{210} \mathrm{~Pb}$ from PTFE reflector; ${ }^{125} \mathrm{I},{ }^{121} \mathrm{Te},{ }^{121 m} \mathrm{Te},{ }^{123 m} \mathrm{Te},{ }^{125 m} \mathrm{Te},{ }^{127 m} \mathrm{Te},{ }^{113} \mathrm{Sn}$, ${ }^{109} \mathrm{Cd},{ }^{22} \mathrm{Na}$, and ${ }^{3} \mathrm{H}$ for cosmogenic isotopes, and have fitted them to the data to estimate their unknown contamination levels.

In Fig. 7 we compare the fitted activities of internal ${ }^{40} \mathrm{~K}$ and ${ }^{210} \mathrm{~Pb}$ to their measured levels for the six crystals, where there is agreement at the $\sim 20 \%$ level. The fitted activities of cosmogenic isotopes: ${ }^{3} \mathrm{H},{ }^{109} \mathrm{Cd},{ }^{22} \mathrm{Na}$, and ${ }^{125} \mathrm{I}$, surface ${ }^{210} \mathrm{~Pb}$, and PTFE ${ }^{210} \mathrm{~Pb}$ for the six crystals are shown in Fig. 8. As explained in Sect. 4, Crystal-1 and 4 have twoyear-long exposure times and, thus, relatively high ${ }^{3} \mathrm{H}$ levels (see Fig. 8a). The largest contribution of ${ }^{109} \mathrm{Cd}$ is in Crystal-4 (Fig. 8b) and the fitted activity is in a good agreement with the measured value. The fitted activities of ${ }^{125} \mathrm{I}$ in Crystal-4, 6, and 7 are similar to each other and higher than other crystals due to their relatively short cooling times underground, as shown in Fig. 8c. There are more contributions from surface ${ }^{210} \mathrm{~Pb}$ in Crystal-1, 2, and 3, and small contributions from PTFE ${ }^{210} \mathrm{~Pb}$ in Crystal-1 and 2. Crystal-3 has large contributions from the surface ${ }^{210} \mathrm{~Pb}$ for both the crystal itself and the PTFE reflector, albeit with large errors.

Based on the background model for the six $\mathrm{NaI}(\mathrm{Tl})$ detectors we determine the background levels in units of dru (counts/day $/ \mathrm{keV} / \mathrm{kg}$ ) in the $2-6 \mathrm{keV}$ energy interval and list them in Table 4. The dominant background contributions are from ${ }^{210} \mathrm{~Pb}$ and ${ }^{3} \mathrm{H}$; representative background spectra of Crystal-7 in the 2-6 keV energy region are shown in Fig. 9. The overall simulated background spectra well describe the measured data for the six $\mathrm{NaI}(\mathrm{Tl})$ detectors.

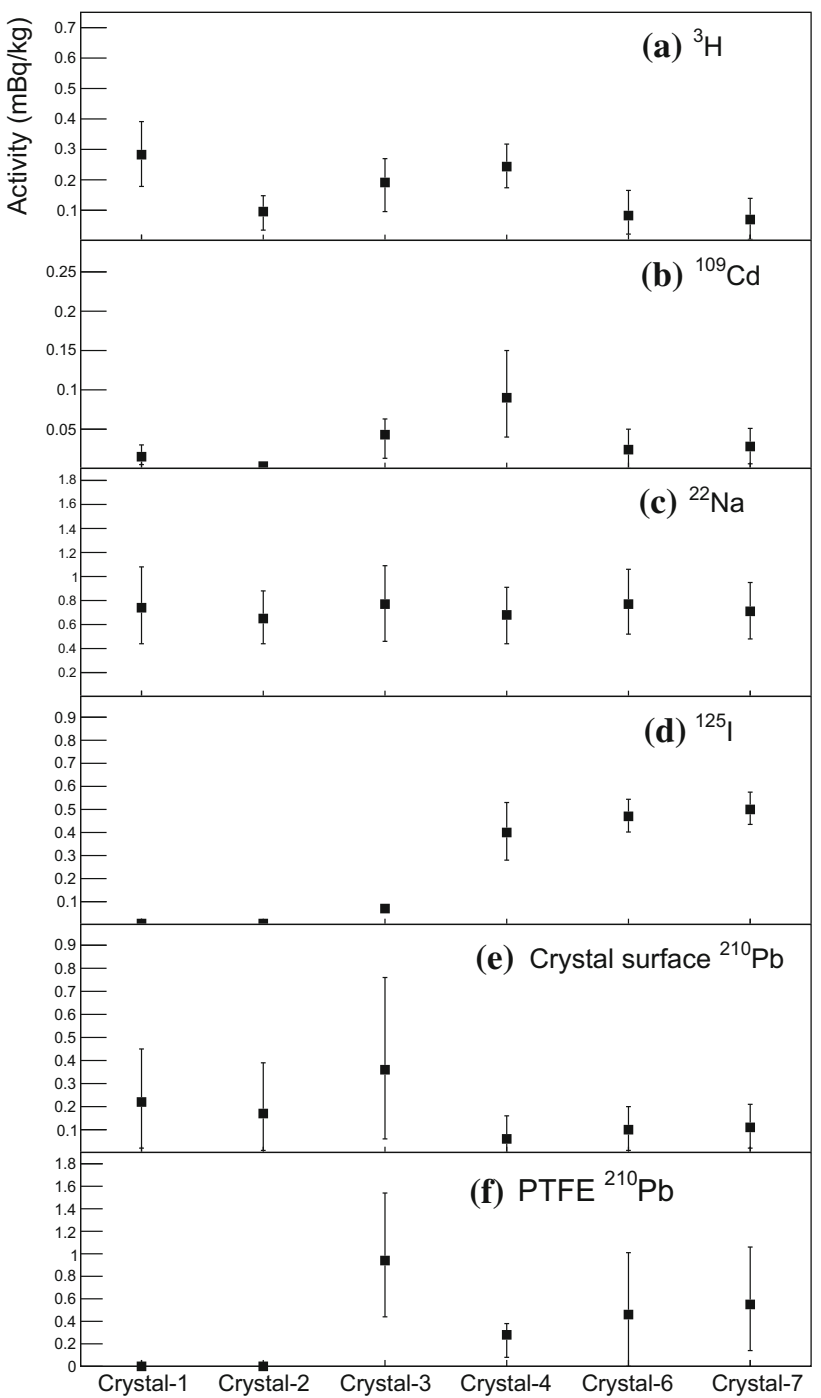

Fig. 8 Fitted activities for $\mathbf{a}^{3} \mathrm{H}, \mathbf{b}{ }^{109} \mathrm{Cd}, \mathbf{c}^{22} \mathrm{Na}, \mathbf{d}{ }^{125} \mathrm{I}$, e Crystal surface ${ }^{210} \mathrm{~Pb}$, and $\mathbf{f} \mathrm{PTFE}{ }^{210} \mathrm{~Pb}$ that correspond to the averaged activities between Oct 21 and Dec 19 2016. Activities of crystal surface ${ }^{210} \mathrm{~Pb}$ and PTFE ${ }^{210} \mathrm{~Pb}$ were normalized by a total mass of the crystal 
Table 4 Fitted background events in units of dru (counts/day/keV/kg) in the (2-6) keV energy interval

\begin{tabular}{|c|c|c|c|c|c|c|}
\hline & Crystal-1 & Crystal-2 & Crystal-3 & Crystal-4 & Crystal-6 & Crystal-7 \\
\hline \multicolumn{7}{|l|}{ Internal } \\
\hline${ }^{40} \mathrm{~K}$ & $0.10 \pm 0.02$ & $0.20 \pm 0.02$ & $0.10 \pm 0.01$ & $0.10 \pm 0.01$ & $0.05 \pm 0.01$ & $0.05 \pm 0.01$ \\
\hline${ }^{210} \mathrm{~Pb}$ & $2.50 \pm 0.10$ & $1.69 \pm 0.09$ & $0.57 \pm 0.05$ & $0.71 \pm 0.05$ & $1.46 \pm 0.07$ & $1.50 \pm 0.07$ \\
\hline Other $\left(\times 10^{-4}\right)$ & $7.0 \pm 0.1$ & $15 \pm 1$ & $7.3 \pm 0.1$ & $7.7 \pm 0.1$ & $14 \pm 1$ & $14 \pm 1$ \\
\hline \multicolumn{7}{|l|}{ Cosmogenic } \\
\hline${ }^{3} \mathrm{H}$ & $2.35 \pm 0.90$ & $0.81 \pm 0.40$ & $1.54 \pm 0.77$ & $1.97 \pm 0.66$ & $0.69 \pm 0.67$ & $0.58 \pm 0.54$ \\
\hline${ }^{109} \mathrm{Cd}$ & $0.05 \pm 0.04$ & $0.009 \pm 0.009$ & $0.13 \pm 0.06$ & $0.29 \pm 0.15$ & $0.08 \pm 0.08$ & $0.09 \pm 0.09$ \\
\hline Other & - & - & $0.02 \pm 0.01$ & $0.09 \pm 0.04$ & $0.06 \pm 0.03$ & $0.05 \pm 0.03$ \\
\hline \multicolumn{7}{|l|}{ Surface } \\
\hline${ }^{210} \mathrm{~Pb}$ & $0.64 \pm 0.64$ & $0.51 \pm 0.51$ & $1.16 \pm 0.51$ & $0.22 \pm 0.16$ & $0.34 \pm 0.20$ & $0.38 \pm 0.21$ \\
\hline \multicolumn{7}{|l|}{ External } \\
\hline & $0.03 \pm 0.02$ & $0.05 \pm 0.04$ & $0.03 \pm 0.02$ & $0.03 \pm 0.02$ & $0.04 \pm 0.03$ & $0.03 \pm 0.02$ \\
\hline \multicolumn{7}{|l|}{ Total simulation } \\
\hline & $5.68 \pm 1.04$ & $3.28 \pm 0.67$ & $3.57 \pm 0.76$ & $3.41 \pm 0.75$ & $2.74 \pm 0.61$ & $2.70 \pm 0.51$ \\
\hline \multicolumn{7}{|l|}{ Data } \\
\hline & $5.64 \pm 0.10$ & $3.27 \pm 0.07$ & $3.35 \pm 0.07$ & $3.19 \pm 0.05$ & $2.62 \pm 0.05$ & $2.64 \pm 0.05$ \\
\hline
\end{tabular}

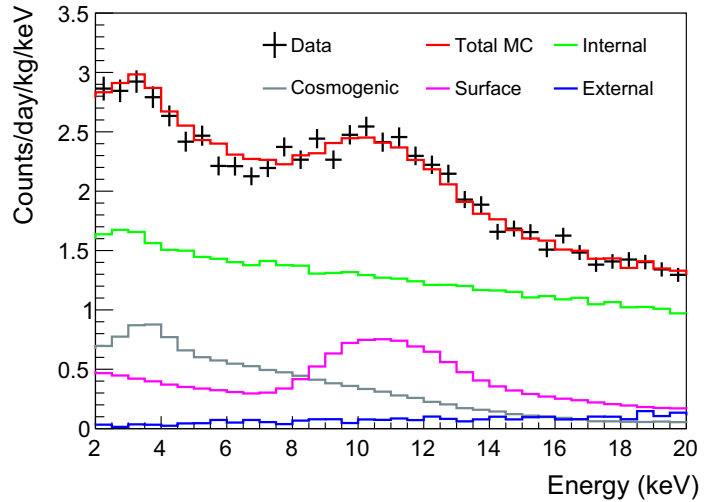

Fig. 9 Comparison of data and Monte Carlo simulation of the lowenergy background spectra of Crystal-7

\section{Conclusion}

We have studied, using the Geant 4 toolkit, the background of the $\mathrm{NaI}(\mathrm{Tl})$ crystal detectors that are being used in the COSINE-100 dark matter search experiment. The crystals have different exposure histories and underground radioactivity cooling times.

In the background modeling the overall energy spectrum summed over all simulations is well matched to the data not only for single-hit events but also for multiple-hit events. Crystal-1 and Crystal-2 that had cooling times as long as three years at the $\mathrm{Y} 2 \mathrm{~L}$ are dominated by ${ }^{210} \mathrm{~Pb}$ and ${ }^{3} \mathrm{H}$ for energies below $20 \mathrm{keV}$. The background contribution of ${ }^{3} \mathrm{H}$ in Crystal-2 is smaller than that in Crystal-1 due to its shorter surface exposure time. Crystal- 6 and 7 show clear contributions from ${ }^{125}$ I due to their short cooling times underground, as expected. Crystal-3 had an additional treatment for a repair that increased the background near $10 \mathrm{keV}$ that is well modeled by surface ${ }^{210} \mathrm{~Pb}$ on the PTFE wrapping foil. Crystal-4 was exposed to surface cosmic rays for two years and only had a six month-long underground cooling time. As a result, this crystal has significant background contributions from both short-lived and long-lived cosmogenic isotopes.

Background contributions from external sources and internal ${ }^{40} \mathrm{~K}$ are reduced to the level of 0.03 dru and about $0.1 \mathrm{dru}$ in the energy range of $2-6 \mathrm{keV}$, respectively, by the LS veto detector that surrounds the crystals.

The average background rate in the (2-6) keV energy range for the six crystals (with a total mass of $70 \mathrm{~kg}$ ) studied here is 3.5 counts $/ \mathrm{day} / \mathrm{keV} / \mathrm{kg}$. The dominant contributions in this energy range are from ${ }^{210} \mathrm{~Pb}$ and ${ }^{3} \mathrm{H}$.

Acknowledgements We thank the Korea Hydro and Nuclear Power (KHNP) Company for providing underground laboratory space at Yangyang. This work is supported by: the Institute for Basic Science (IBS) under project code IBS-R016-A1, Republic of Korea; UIUC campus research board, the Alfred P. Sloan Foundation Fellowship, NSF Grants no. PHY-1151795, PHY-1457995, DGE-1122492 and DGE1256259, WIPAC, the Wisconsin Alumni Research Foundation, Yale University and DOE/NNSA Grant no. DE-FC52-08NA28752, United States; STFC Grant ST/N000277/1 and ST/K001337/1, United Kingdom; and CNPq and Grant no. 2017/02952-0 FAPESP, Brazil.

Open Access This article is distributed under the terms of the Creative Commons Attribution 4.0 International License (http://creativecomm ons.org/licenses/by/4.0/), which permits unrestricted use, distribution, and reproduction in any medium, provided you give appropriate credit to the original author(s) and the source, provide a link to the Creative Commons license, and indicate if changes were made. Funded by SCOAP ${ }^{3}$. 


\section{References}

1. K.W. Kim et al. (KIMS Collaboration). Astropart. Phys. 62, 249 (2015)

2. P. Adhikari et al. (KIMS Collaboration). Eur. Phys. J. C 76, 185 (2016)

3. R. Bernabei et al., Eur. Phys. J. C 56, 333 (2008)

4. R. Bernabei et al., Eur. Phys. J. C 67, 39 (2010)

5. R. Bernabei et al., Eur. Phys. J. C 73, 2648 (2013)

6. R. Bernabei et al. (DAMA/LIBRA). arXiv:1805.10486

7. G. Adhikari et al., Eur. Phys. J. C 78, 107 (2018)

8. E. Barbosa de Souza et al. (DM-Ice Collaboration). Phys. Rev. D 95, 032006 (2017)

9. J. Amare et al., Nucl. Instrum. Meth. Phys. Res., Sect. A 742, 187 (2014)

10. J. Amare et al., Eur. Phys. J. C 76, 429 (2016)

11. J. Cherwinka et al. (DM-Ice Collaboration). Phys. Rev. D 90, 092005 (2014)

12. K. Fushimi et al., Phys. Proc. 61, 67 (2015)

13. C. Tomei et al. (SABRE Collaboration). Nucl. Instrum. Meth. A 845, 418 (2017)
14. G. Angloher et al., Eur. Phys. J. C 76, 441 (2016)

15. H.S. Lee et al. (KIMS Collaboration). Phys. Rev. Lett. 99, 091301 (2007)

16. S.C. Kim et al. (KIMS Collaboration). Phys. Rev. Lett. 108, 181301 (2012)

17. S. Agostinelli et al., Nucl. Instrum. Meth. Phys. Res., Sect. A 506, 250 (2003)

18. Alpha Spectra Inc. http://www.alphaspectra.com

19. S. Chauvie et al., Conf. Rec. IEEE Nucl. Sci. Symp., pp. 1881-1885 (2004)

20. Tullio Basaglia et al., J. Phys.: Conf. Ser. 664, 072037 (2015)

21. J. Allison et al., Nucl. Instrum. Meth. Phys. Res., Sect. A 835, 186 (2016)

22. G. Adhikari et al., Eur. Phys. J. C 77, 437 (2017)

23. M. Lee et al., J. Kor. Phys. Soc. 58(4), 713-718 (2011)

24. Walter C. Pettus, Ph.D. thesis, University of Wisconsin-Madison (2015)

25. J. Amare et al., JCAP 02, 046 (2015)

26. P. Villar et al., Int. J. Mod. Phys. A 33(9), 1843006 (2018)

27. J. Amare et al. Astropart. Phys. 97, 96 (2018) 